\title{
Frecuencia y procedencia del antígeno Kell en mujeres donantes de sangre durante los años 2016-2017
}

Fonseca Joya Marghy Daniela. Programa Bacteriología y Laboratorio Clínico; Universidad de Boyacá, Tunja, Colombia.

Murcia-Alarcón Ángela Constanza Programa Bacteriología y Laboratorio Clínico; Universidad de Boyacá, Tunja, Colombia.

Pardo-Reyes Yuli Paola. Programa Bacteriología y Laboratorio Clínico; Universidad de Boyacá, Tunja, Colombia.

Cruz-Rubio Shirley Bacterióloga y Laboratorista Clínica Mg, Especialista en hematología y banco de sangre, Universidad de Boyacá, Tunja, Colombia; Grupo de Investigación del programa de Bacteriología y Laboratorio Clínico, Universidad de Boyacá, Tunja, Colombia.

Merchan Nuri Andrea. Microbiologa industrial, Doctorado en biotecnología, Universidad de Boyacá, Tunja, Colombia; Grupo de Investigación del programa de Bacteriología y Laboratorio Clínico, Universidad de Boyacá, Tunja, Colombia.

Fecha de recepción: 07/07/2018

Fecha de corrección: 30/01/2019

Fecha de aprobación 02/18/2019

Fecha de publicación 30/06/2019

\section{Resumen}

Introducción: El sistema Kell está formado por dos antígenos principales: el Kell $(\mathrm{K})$ y el Cellano $(\mathrm{k})$, estos son capaces de causar reacciones graves, tales como reacción hemolítica postransfusional y la enfermedad hemolítica del recién nacido. Los antígenos de este sistema son altamente inmunogénicos lo que les confiere el tercer lugar en importancia clínica.

Objetivo: Determinar la frecuencia del antígeno Kell y procedencia de las mujeres donantes de sangre con antígeno Kell positivo en el Hemocentro del Centro Oriente Colombiano (HCOC).

Metodología: Estudio descriptivo de corte transversal que incluyó 186 donantes voluntarias de sangre del Hemocentro Centro Oriente Colombiano, se realizó la fenotipificación del antígeno Kell, utilizando la técnica Aglutinación en lámina, la cual se basa en enfrentar glóbulos rojos del donante con anticuerpo monoclonal anti K. Se calculó la frecuencia fenotípica del antígeno Kell, en porcentajes y para el procesamiento de la información se utilizó el paquete estadístico SPSS versión 21.0 en español donde se realizó todo el análisis de los datos de la población.

Resultados: Se procesaron 177 muestras obtenidas en 9 campañas de donación de sangre realizadas en diferentes municipios del departamento de Boyacá, obteniéndose una frecuencia fenotípica del $7,5 \%$ para el antígeno Kell, en la población de mujeres donantes de sangre del HCOC, siendo esta similar con la frecuencia encontrada en Colombia y Latinoamérica.

Conclusión: Se determinó que la frecuencia del antígeno Kell en las mujeres donantes de sangre del HCOC fue del 7,5\%, y se logró identificar que no existe una relación estadísticamente entre la procedencia y la presencia del antígeno Kell en las donantes, lo anterior está relacionado con el mestizaje y los procesos de migración.

Palabras claves: eritroblastosis fetal, Sistema del Grupo Sanguíneo de Kell, Complejo Antígeno-Anticuerpo.

Copyright (C) Facultad de Ciencias de la Salud de la Universidad Tecnológica de Pereira. 1995-2018. Todos los derechos reservados.
Frequency and origin of the Kell antigen in female blood donors during 2016-2017

\begin{abstract}
Introduction: The Kell system consists of two major antigens: Kell $(\mathrm{K})$ and Cellano $(\mathrm{K})$, which are capable of causing serious reactions, such as posttransfusion hemolytic reaction and hemolytic disease of the newborn. The antigens of this system are highly immunogenic which gives them the third place in clinical importance.
\end{abstract}

Objective: To determine the frequency of Kell antigen and origin of blood donors in the Hemocenter of the Centro Oriente Colombiano (H.C.O.C).

Methods: Cross-sectional descriptive study involving 186 blood donors from the Centro Oriente Colombian Hemocenter, phenotyping of the Kell antigen was carried out, using the technique Aglutination in lamina, which is based on facing donor red blood cells with anti-K monoclonal antibody. Calculated the phenotypic frequency of the Kell antigen in percentages and for the processing of the information was used the statistical package SPSS version 21.0 in Spanish where all the analysis of the data of the population was carried out.

Results: 177 samples obtained in 9 blood donation campaigns were carried out in different municipalities of the department of Boyacá, obtaining a phenotypic frequency of $7.5 \%$ for the Kell antigen in the population of female HCOC blood donors. Similar to the frequency found in Colombia and Latin America.

Conclusion: It was determined that the frequency of Kell antigen in the female HCOC donors was $7.5 \%$, and it was possible to identify that there is no statistically relation between the origin and the presence of Kell antigen in the donors, Is related to mestizaje and migration processes.

Key words: fetal erythroblastosis, Kell Blood-Group System, Antigen-Antibody Complex.

Copyright (C) Facultad de Ciencias de la Salud de la Universidad Tecnológica de Pereira. 1995-2018. Todos los derechos reservados. 


\section{Introducción}

El sistema Kell está formado por 32 antígenos, los principales son dos: el Kell (K1) y el Cellano (k2), el primero fue descubierto en 1946 por Coombs, Mourant y Race cuando encontraron el anticuerpo respectivo en un caso de enfermedad hemolítica del recién nacido. Tres años después Levine, encontraron el alelo respectivo, el cual fue denominado Cellano [1].

Los antígenos de este sistema son altamente inmunogénicos, lo que les confiere el tercer lugar en importancia clínica después de ABO y $\mathrm{Rh}$, ya que producen anemia por dos mecanismos: hemólisis de los glóbulos rojos o por una inhibición de los precursores eritropoyéticos a nivel de la médula ósea fetal se encuentran en la superficie de los glóbulos rojos humanos y están completamente desarrollados al nacimiento [2].

La glicoproteína que codifica para el gen Kell se localiza en el brazo largo del cromosoma 7, en la posición 7q 33. Este gen codifica una proteína glicosilada de 93 kilodaltons la cual tiene áreas específicas donde se expresan varios antígenos Kell, de una manera similar de como lo hacen los antígenos del sistema Rh. El antígeno $\mathrm{K}$ es heredado en forma mendeliana, codominante con su alelo k. Los distintos polimorfismos del sistema Kell se producen por la sustitución de una base nucleotídica que conduce a un cambio de aminoácido en la glicoproteína Kell, El polimorfismo Kell/Cellano ocurre en Thr193Met [3].

Anti-K y anti k son capaces de causar reacciones graves, tales como reacción hemolítica pos transfusional y la enfermedad hemolítica del recién nacido (EHRN) [4].

La EHRN es una afección inmunológica aloinmune, mediada por anticuerpos maternos dirigidos contra antígenos de origen paterno presentes en los hematíes fetales. Estos anticuerpos de la madre atraviesan la placenta, sensibilizan los hematíes fetales y provocan hemólisis de los glóbulos rojos. En casos muy graves, el feto puede desarrollar hidropesía fetal y morir en el útero por falla cardiaca congestiva, secundaria a la anemia hemolítica $[5,6]$. La EHRN fue considerada hace unas décadas una enfermedad frecuente y grave que influía considerablemente en la morbimortalidad perinatal, en la actualidad es una patología de aparición ocasional cuya incidencia puede estimarse en 1 por cada 1000 nacidos vivos [7].

La distribución de la frecuencia fenotípica y genotípica para ambos sistemas sanguíneos son similares en diversas poblaciones del mundo; el antígeno k2 es el más frecuente $(99,5 \%)$ y K1 $(4 \%)$ [8]. La frecuencia alta o baja de un antígeno eritrocitario cobra relevancia cuando se tienen receptores de sangre que previamente se hayan aloinmunizados contra ellos, pues si el aloanticuerpo reconoce un antígeno de alta frecuencia, como lo es el antígeno k2, será muy complejo encontrar glóbulos rojos compatibles [9].

\section{Materiales y métodos}

Población de estudio: Se realizó un estudio descriptivo de corte transversal. La población en estudio correspondió a 186 donantes de sangre voluntarias que acudieron a colectas móviles organizadas por el Hemocentro Centro Oriente Colombiano (H.C.O.C) en diferentes municipios del departamento de Boyacá. Esta muestra fue establecida por la calculadora stat-calc del programa estadístico Epiinfo ver 7.0 con los siguientes criterios: nivel de confianza del $95 \%$, proporción esperada del $90 \%$ y un total de población de 359 mujeres donantes correspondientes al promedio mensual de unidades recolectadas de mujeres en H.C.O.C durante el año 2015. Como criterios de exclusión se tuvo en cuenta ser de sexo masculino, donantes Rh negativas con fenotipificación de antígeno Kell por H.C.O.C

Muestra de sangre: La muestra fue recolectada de la tubulatura central de la unidad fraccionada de glóbulos rojos, para realizar la fenotipificación del antígeno Kell. Las participaron diligenciaron consentimiento informado y el trabajo contó con el aval del comité de bioética de la Facultad de ciencias de la salud.

Fenotipificación del antígeno Kell: se realizó mediante la técnica de aglutinación en lámina, la cual se basa en enfrentar glóbulos rojos del donante con anticuerpo monoclonal anti k. La aglutinación o no de los hematíes frente al reactivo indica la presencia o ausencia del antígeno Kell en la superficie de la membrana de los glóbulos rojos. Para la interpretación de resultados se tuvo en cuenta la aglutinación que se evidencio macroscópicamente indicando si es positivo o negativo.

\section{Resultados}

Se identificó el fenotipo de acuerdo con el patrón de reacción obtenido al realizar los análisis de cada muestra, dando como resultado final una frecuencia del 7,5\% $(\mathrm{n}=14)$ muestras positivas, $87,6 \%(\mathrm{n}=163)$ muestras negativas y un $4,8 \%(\mathrm{n}=9)$ de las muestras no se procesaron, debido a que 8 muestras fueron reactivas para alguno de los siete marcadores infecciosos y una por control de calidad del HCOC.

Para establecer la procedencia de las donantes con antígeno Kell positivo que acudieron al HCOC se tuvo en cuenta el lugar de nacimiento dato que se consiguió mediante la encuesta otorgada por el HCOC y se evidenció que las participantes proviene dentro y fuera del departamento de Boyacá, debido a la migración hacia esta región que es donde se llevó a cabo el presente estudio (figura 1), lo anterior permite deducir que este antígeno se puede encontrar en donantes de cualquier municipio de la región o del departamento de Boyacá.

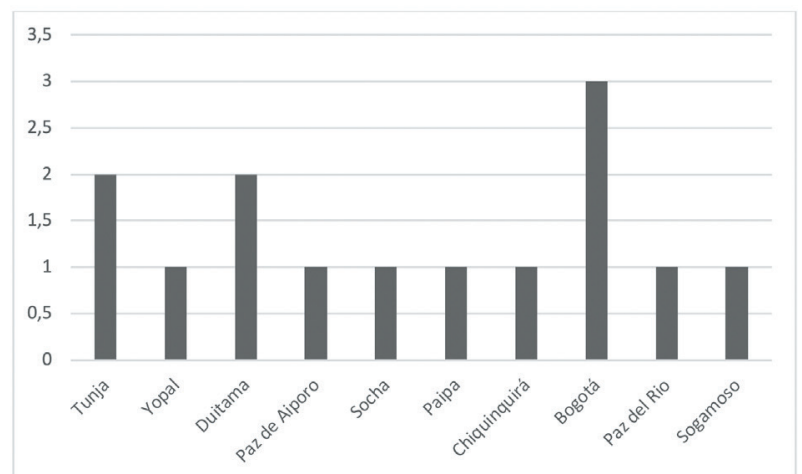

Figura 1 Procedencia de las donantes con antígeno Kell positivo que acudieron al HCOC.

\section{Discusión.}

Este estudio ha permitido conocer la distribución de frecuencia fenotípica del antígeno Kell en donantes voluntarias del H.C.C.O.C del departamento de Boyacá. Cabe mencionar que en Colombia especialmente en el departamento de Boyacá no se han realizado estudios sobre antígeno Kell, lo que nos permite realizar la caracterización fenotípica en la población, permitiendo evaluar las variaciones o similitudes con otras poblaciones.

El sistema sanguíneo Kell es de alto interés conocer la frecuencia y de sus principales antígenos (K1 y k2) en la población, por ser el tercero en importancia clínica. La frecuencia del antígeno Kell (7,5\%) se asemeja a los encontrados en la población de Santiago de Chile en el año 1988 en que la frecuencia para K fue del 4\%. De igual tendencia se informa un estudio de Chargoy en que se detectaron frecuencias para el antígeno $\mathrm{K}$ del $2 \%$, la principal conclusión de ese estudio es que esta tendencia es debido a la mezcla genómica de los pueblos aborígenes con los españoles como resultado de la colonización del siglo XVI [10].

De igual forma, en diversos textos se reparten frecuencias en poblaciones que fluctúan del 2 al 9\% para el antígeno K1 del 91 al $99 \%$ para el antígeno k2 [11]. En estudios más actuales realizados 
en la población palestina, se reportaron frecuencias del antígeno K1 del 5,63\%; y para el k2, 94,4\% [12]. En las localidades de guajarat y delhi, india, los resultados de frecuencia fueron para el K1 del $6,09 \%$ y $3,5 \%$; y k2 del $100 \%$ y $99,97 \%$ respectivamente. Resultados similares a la frecuencia del estudio para K1 [13].

El estudio es realizado sobre frecuencia de antígenos del sistema sanguíneo $\mathrm{Rh}$ y del sistema Kell en donantes de sangre de la región del Maule en Chile en el año 2015, con una muestra de 200 donantes, reportaron una frecuencia de 4\% para K1 y k2 del 95\%, lo que significa que en el estudio que realizamos la frecuencia que se asemeja es la de K1 [14].

Un estudio realizado en Colombia para antígeno Kell en la ciudad de Bogotá en el año 2012 con 97 donantes se evidencio una frecuencia para Kell del 4,1\%, lo que representa una frecuencia similar a la obtenida en el presente estudio [15].

En la población de mujeres donantes de sangre del HCOC la frecuencia fenotípica para el antígeno Kell fue similar con la de poblaciones estudiadas como por ejemplo, en Guatemala por Alvarado y Dubón (2012) y en Colombia por el grupo de Dueñas (1999) en Cali donde encontraron una frecuencia de 4.8 de anti K en un grupo de donantes de sangre. A pesar de ser una frecuencia baja, es importante la fenotipificación del antígeno Kell, ya que este es el más inmunogénico luego del antígeno D.

La presencia de anti-K, se encuentra directamente relacionada con la etiopatogenia de la enfermedad hemolítica del recién nacido y asociados con la sensibilización por embarazo. También se refleja la vulnerabilidad de la historia de embarazos y transfusión para prevenir las reacciones por anticuerpo inesperados como es anti-K.

No existe una relación entre la procedencia y la presencia de antígeno Kell, asociado principalmente al mestizaje y a la migración de la población colombiana.

Para el HCCOC es importante contar con una base de datos de donantes de sangre fenotipificados, que permite suministrar unidades de glóbulos rojos compatibles para personas que previamente se han aloinmunizado o idealmente prevenir la inmunización en individuos que requieran ser poli transfundidos como los pacientes hematoncológicos o las niñas, adolescentes y mujeres en edad fértil.

Conflictos de interés: Los autores declaran que no tienen conflictos de interés

\section{Referencias}

1. Zacarias JM, Lnager IB, Vicentainer JM. Profile of Rh, Kell, Duffy, Kidd, and Diego Blood Group Systems among Blood Donors in the Southwest region of the Parana state, Southern Brazil. Rev Transfus Apher Sci 2016;55(3):302-307. Available from: https://www.ncbi.nlm.nih.gov/pubmed/27566949.

2. Zalpuri S, Middelburg RA, Schonewille H, De Vooght KMK, Le Cessie S, Van Der Bom JG, et al. Intensive red blood cell transfusions and risk of alloimmunization. Rev Transfusion 2014;54(2):278-84. Available from: https://www.ncbi.nlm.nih. gov/pubmed/23782244.

3. Sankaralingam P, Jain A, Bagga R, Kumar P, Marwaha N. Red cell alloimmunization in RhD positive pregnant women and neonatal outcome. Rev Transfusion and Apheresis Science 2016;55(1):153-158. Available from: https://www.ncbi.nlm.nih. gov/pubmed/27324408.

4. Krishna D, Babu S, Vijaya K, Rajendran A, Sarella JD. Prevalence of Principal Rh Blood Group Antigens in Blood Donors at the Blood Bank of a Tertiary Care Hospital in. Rev Journal of Clinical and Diagnostic Research 2016; 10(5) 7-10. Available from: https://www.ncbi.nlm.nih.gov/pmc/articles/ PMC4948399/pdf/jcdr-10-EC07.pdf
5. Brumbaugh JE, Morgan S, Beck JC, Zantek N, Kearney S, Bendel CM, et al. Blueberry muffin rash, hyperbilirubinemia , and hypoglycemia: A case of a hemolytic disease of the fetus and newborn due to anti-Kp. Journal of Perinatology 2011;31(5):373-6. Available from: http://dx.doi.org/10.1038/ jp. 2010.161

6. Stowell SR, Henry KL, Smith NH, Hudson KE, Halverson GR, Park JC, et al. Alloantibodies to a paternally derived RBC KEL antigen lead to hemolytic disease of the fetus/newborn in a murine model. Blood 2013;122(8):1494-504.

7. Makarovska, Blagoevska M, Kolevski P, Kostovska S. Optimal Blood Grouping And Antibody Screening for safe transfusion. Rev Contributions of Macedonian Academy of Sciences \& Arts 2009; 30(1):119-128. Available from: https://www.ncbi.nlm. nih.gov/pubmed/19736535

8. Fuenzalida C J, Carvajal C JA. Manejo de la embarazada con isoinmunización por anticuerpos irregulares. Rev Chil Obstet Ginecol 2014;79(4):315-22. Available from: http:// www.scielo.cl/scielo.php? script $=$ sci arttext\&pid $=$ S0717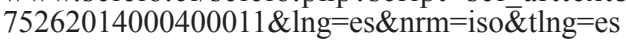

9. Cortey A, Mailloux A, Huguet-Jacquot S, Castaigne-Meary V, Macé G, Guyen A. Incompatibilidad eritrocítica maternofetal. Rev Scien Direc 2012;47(4):1-22. Available from: http:// linkinghub.elsevier.com/retrieve/pii/S1245178912635227.

10. Chargoy-Vivaldo, Azcona-Cruz R-A. Prevalencia del antígeno Kell $(\mathrm{K}+)$ en muestras obtenidas en un banco de sangre. Rev Hematol 2016;17(2):114-22. Available from: http://new. medigraphic.com/cgi-bin/resumen.cgi?IDARTICULO $=67043$.

11. Paulina AUL. Análisis retrospectivo de la frecuencia y tipo de anticuerpos irregulares en donantes voluntarios de sangre en el hemocentro de la cruz roja ecuatoriana, quito. Hematologia. Pontificia Universidad Catolica del Ecuador 2009-2012.

12. Tarkla B, Saluja K, Sam R .Phenotype frequencies of blood group systems (Rh, Kell, Kidd, Duffy, MNS, P, Lewis, and Lutheran) in north Indian blood donors. Rev Transfusion and apheresis science 2010;43(1):17-22. Available from: http:// www.trasci.com/article/S1473-0502(10)00089-3/abstract

13. Makroo RN, Bhatia A, Gupta R, Phillip J. Prevalence of Rh, Duffy, Kell, Kidd \& MNSs blood group antigens in the Indian blood donor population. The Indian Journal of Medical Research. 2013;137(3):521-6. Available from: https://www. scienceopen.com/document?vid=aae8e69d-3da2-4bee-8fae5d932b698a79\&sort=5

14. Vasques Rojas M, Castillo D, Pavez Y. Frecuencia de antígenos del sistema sanguíneo $\mathrm{Rh}$ y del sistema Kell en donantes de sangre. Rev Cuba Hematol Inmunol Hemoter. 2015;31(2):160 71.

15. Barragán LE, Lucía $\mathrm{O}$, Vargas J, Isaza M. Prevalencia de anticuerpos irregulares contra antígenos eritrocitarios en gestantes que asisten al control prenatal en una clínica de Bogota Colombia. 2012;100(3). Available from: http://www.aahi.org.ar/ PostersCongreso2013/E-7.pdf 